\title{
Drawing Methods of Output Force Distribution by Measurement of Sides
}

\author{
Satoshi Komada ${ }^{* a)}$ Senior Member, Shohei Ishida* Non-member

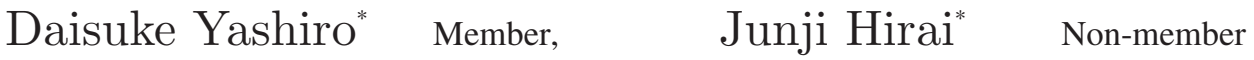 \\ Akinobu Nishimura** Non-member
}

(Manuscript received March 25, 2015, revised June 22, 2015)

\begin{abstract}
The theory of functionally different effective muscles allows for detailed measurement of the muscular strength of limbs by separating mono-articular muscles and bi-articular muscles. The theory represents the output force distribution (OFD) at the end of a limb as a hexagon whose opposite sides are parallel and the same length. Because the OFD is divided into independent muscular strengths, it must be measured accurately. The OFD can be drawn from measurements of five of the six sides. This paper proposes a method to draw the OFD from measurements of all six sides. Because the measurement does not satisfy the characteristics of the OFD, an optimization problem is proposed for minimizing the distance from the original measurement while satisfying the characteristics. These two drawing methods of OFD were applied to the lower limbs of three subjects for three different postures. Because the error of the five-side measurement method is dependent on the estimated side and posture of lower limbs, the estimated side that realizes the smallest error of the OFD for a given posture can be determined from geometrical analysis. The OFD error of the optimization method was about half that of the five-side measurement method.
\end{abstract}

Keywords: limb, functionally different effective muscle, output force distribution, measurement of sides, optimization

\section{Introduction}

General muscular strength measurement of limbs uses joint torque measurement devices. It is impossible to obtain individual muscular strength because the joint torque is sum of the muscular strength related to its joint.

Functionally different effective muscle (FEM) has been proposed, where muscles of limbs are classified into three antagonist pairs of six muscle groups according to influence to joints in the plane made by limbs, which represents relation between torque of each muscle group and corresponding output force at the end of a limb ${ }^{(1)}$. Maximum range of force at the end of a limb referred to as output force distribution (OFD) is defined as hexagon having geometrical characteristics. Individual maximum muscular strength is evaluated by measured OFD. The FEM theory has been applied to evaluation of driving force of wheelchairs ${ }^{(2)}$, development of a steering wheel system ${ }^{(3)}$, strength training ${ }^{(4)}$, and so on.

The accuracy of the muscular strength evaluation method depends on measurement accuracy of OFD. The four-point measurement method has to measure one vertex of OFD whose direction varies depending on muscular strength ${ }^{(5)}$.

a) Correspondence to: Satoshi Komada. E-mail: komada@elec. mie-u.ac.jp

* Department of Electrical and Electronic Engineering, Graduate School of Engineering, Mie University

1577, Kurimamachiya, Tsu, Mie 514-8507, Japan

** Department of Orthopaedic and Sports Medicine, Graduate

School of Medicine, Mie University

2-174, Edobashi, Tsu, Mie 514-8507, Japan
Because searching for the vertex is difficult, it affects accuracy of OFD. The five-side measurement method improved repeatability of OFD measurement by avoiding the vertex measurement ${ }^{(6)}$. Because the method draws the rest one side using the characteristics of OFD, measurement of one side of OFD which is difficult for subjects to exert force at the end of a limb can be omitted. However, the shapes of OFD are different depending on the side without measurement when knee joint is stretched. Although the problem can be solved if all six sides are measured, the derived hexagon does not satisfy the characteristics of OFD.

Therefore, a drawing method of OFD similar to the hexagon by the six-side measurement through an optimization problem under constraint of the OFD characteristics has been proposed ${ }^{(7)(8)}$. Evaluation of the five-side measurement method and the optimization method was performed using three subjects for two postures of lower limbs. This paper increases measurement postures and analysis between posture of limbs and error of estimated sides by the five-side measurement method.

\section{Theory of Functionally Different Effective Muscles}

2.1 Functionally Different Effective Muscular Strength Relation between force at the end of a limb and muscular strength in 2D space can be defined by the three antagonistic pair model as shown in Fig. $1^{(1)}$. e1 and $\mathrm{f} 1$ are mono-articular muscles affecting hip or shoulder joint J1. e2 and $\mathrm{f} 2$ are mono-articular muscles affecting knee or elbow joint $\mathbf{J} 2$. e3 and $\mathrm{f} 3$ are bi-articular muscles affecting both 

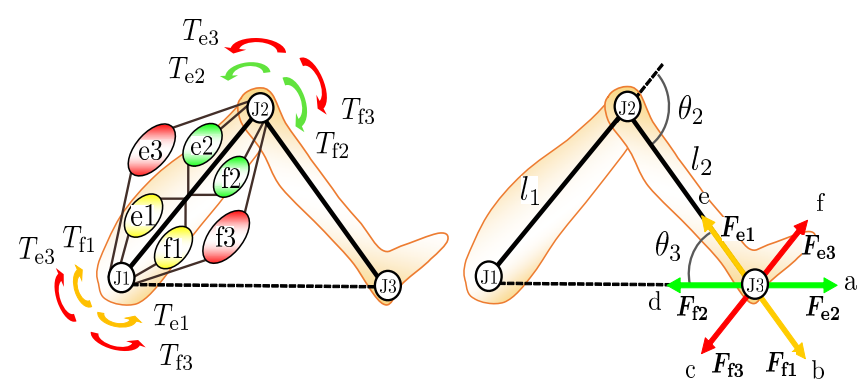

Fig. 1. Functionally different effective muscular strength and corresponding output force at the end of a limb

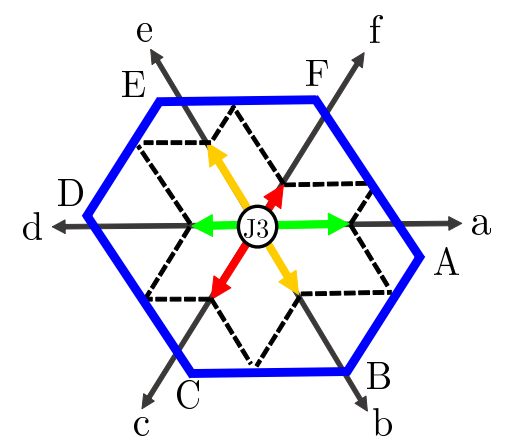

Fig. 2. Output force distribution (OFD)

joints. $T_{\mathrm{e} 1}, T_{\mathrm{e} 2}, T_{\mathrm{e} 3}, T_{\mathrm{f} 1}, T_{\mathrm{f} 2}, T_{\mathrm{f} 3}$ shown in Fig. 1 are joint torque exerted by corresponding FEM.

2.2 Output Force at the End of a Limb The force at the end of a limb J3 caused by the FEM is called end output force. The magnitude of the end output force is decided by

$$
\begin{aligned}
\left|\boldsymbol{F}_{\mathrm{e} 1}\right|=\frac{T_{\mathrm{e} 1}}{l_{1} \sin \theta_{2}}, & \left|\boldsymbol{F}_{\mathrm{f} 1}\right|=\frac{T_{\mathrm{f} 1}}{l_{1} \sin \theta_{2}} \\
\left|\boldsymbol{F}_{\mathrm{e} 2}\right|=\frac{T_{\mathrm{e} 2}}{l_{2} \sin \theta_{3}}, & \left|\boldsymbol{F}_{\mathrm{f} 2}\right|=\frac{T_{\mathrm{f} 2}}{l_{2} \sin \theta_{3}} \\
\left|\boldsymbol{F}_{\mathrm{e} 3}\right|=\frac{T_{\mathrm{e} 3}}{l_{2} \sin \theta_{2}}, & \left|\boldsymbol{F}_{\mathrm{f} 3}\right|=\frac{T_{\mathrm{f} 3}}{l_{2} \sin \theta_{2}}
\end{aligned}
$$

$\boldsymbol{F}_{\mathrm{e} 1}, \boldsymbol{F}_{\mathrm{e} 2}, \boldsymbol{F}_{\mathrm{e} 3}, \boldsymbol{F}_{\mathrm{f} 1}, \boldsymbol{F}_{\mathrm{f} 2}, \boldsymbol{F}_{\mathrm{f} 3}$ are end output force vectors of corresponding FEM. $l_{1}$ and $l_{2}$ are length between J1 and J2 and between $\mathbf{J} 2$ and $\mathbf{J} 3$, respectively. $\theta_{2}$ is bending angle of J2. $\theta_{3}$ is the angle between the line J1-J3 and the line J2-J3. Direction of the end output force is defined by

- $\boldsymbol{F}_{\mathrm{f} 1}, \boldsymbol{F}_{\mathrm{e} 1}: \overrightarrow{\mathrm{eb}}$ and $\overrightarrow{\mathrm{be}}$ directions parallel to line J2-J3

- $\boldsymbol{F}_{\mathrm{f} 2}, \boldsymbol{F}_{\mathrm{e} 2}: \overrightarrow{\mathrm{ad}}$ and $\overrightarrow{\mathrm{da}}$ directions parallel to line J1-J3

- $\boldsymbol{F}_{\mathrm{f} 3}, \boldsymbol{F}_{\mathrm{e} 3}: \overrightarrow{\mathrm{fc}}$ and $\overrightarrow{\mathrm{cf}}$ directions parallel to line J1-J2

2.3 Output Force Distribution By adding the end output force vectors, the hexagonal shape OFD which shows orientation and magnitude of maximum output force as shown in Fig. 2 is obtained. The obtained OFD has the following characteristics.

$$
\begin{array}{ll}
\left|\boldsymbol{F}_{\mathrm{f} 1}\right|+\left|\boldsymbol{F}_{\mathrm{e} 1}\right|=\overline{\mathrm{AF}}=\overline{\mathrm{CD}}, & a_{\mathrm{AF}} / / a_{\mathrm{CD}} / / \overrightarrow{\mathrm{be}} \\
\left|\boldsymbol{F}_{\mathrm{f} 2}\right|+\left|\boldsymbol{F}_{\mathrm{e} 2}\right|=\overline{\mathrm{EF}}=\overline{\mathrm{BC}}, & a_{\mathrm{EF}} / / a_{\mathrm{BC}} / / \overrightarrow{\mathrm{ad}} \\
\left|\boldsymbol{F}_{\mathrm{f} 3}\right|+\left|\boldsymbol{F}_{\mathrm{e} 3}\right|=\overline{\mathrm{DE}}=\overline{\mathrm{AB}}, & a_{\mathrm{DE}} / / a_{\mathrm{AB}} / / \overrightarrow{\mathrm{cf}}
\end{array}
$$

where $a_{\mathrm{i}}$ shows slope of side i ( $\left.\mathrm{i}=\mathrm{AB}, \mathrm{BC}, \mathrm{CD}, \mathrm{DE}, \mathrm{EF}, \mathrm{FA}\right)$. The first characteristic shows length of the opposite sides

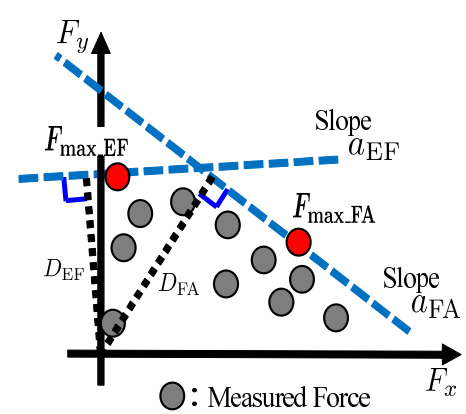

Fig. 3. Side measurement of OFD

is the sum of length of end output force vectors parallel to their sides. The second characteristic shows slope of opposite sides is the same as the slope of end output force vector decided by posture of limbs.

In order to draw OFD, these characteristics must be satisfied.

\section{Drawing of Output Force Distribution by Measurement of Sides}

3.1 Determination Method of Sides Satisfying Parallel Condition The four-point measurement method of OFD requires measurement of one vertex. The measurement is difficult because subjects must find a direction of the vertex which depends on muscular strength ${ }^{(6)}$. In measurement of sides, subjects can exert force to any direction where sides exist.

As shown in Fig. 3, a decision method of each side of OFD from measured end force of limbs $\left(F_{x}, F_{y}\right)$ is given by

$$
\begin{aligned}
& \boldsymbol{F}_{\text {max } \_\mathrm{i}}=\underset{F_{x}, F_{y} \in R}{\arg \max } D_{\mathrm{i}}\left(F_{x}, F_{y}\right) \ldots \ldots \ldots \ldots \ldots \ldots \\
& D_{\mathrm{i}}\left(F_{x}, F_{y}\right)=\frac{\left|b_{\mathrm{i}}\right|}{\sqrt{a_{\mathrm{i}}^{2}+1}}=\frac{\left|F_{y}-a_{\mathrm{i}} F_{x}\right|}{\sqrt{a_{\mathrm{i}}^{2}+1}} \ldots \ldots \ldots \ldots
\end{aligned}
$$

Eq. (3) obtains a measurement point $\boldsymbol{F}_{\text {max_i }}$ whose distance from origin for side i $D_{\mathrm{i}}$ calculated from (4) is the largest. The slope $a_{\mathrm{i}}$ is decided from the posture of limbs to satisfy the characteristic of slope shown in (2). $b_{\mathrm{i}}$ in (4) shows an intercept of the slope $a_{\mathrm{i}}$ line passing through the end force of limbs $\left(F_{x}, F_{y}\right)$. From these equations, farthest measurement points from the origin on sides of OFD can be decided when subjects exert force at the end of a limb.

3.2 Output Force Distribution by Five-side Measurement The five-side measurement method measures five sides among six sides of OFD using (3) and decides the rest one side so that length of opposite sides is the same to fulfill the characteristics of the OFD shown in (2). As shown in Fig. 4 for example, if five sides are measured except for side $\mathrm{CD}$ of an OFD, length of sides EF, FA, and $\mathrm{AB}$ are decided. An OFD is drawn so that each length of the other sides is the same as the opposite sides. Any sides of OFD can be decided by the method without its measurement. The method can delete measurement of a side which is difficult for subjects to exert force. The five-side measurement method realizes measurement of OFD with higher repeatability than the conventional four-point measurement method ${ }^{(6)}$.

3.3 Hexagon by Six-Side Measurement In the five-side measurement method, the following problems are 


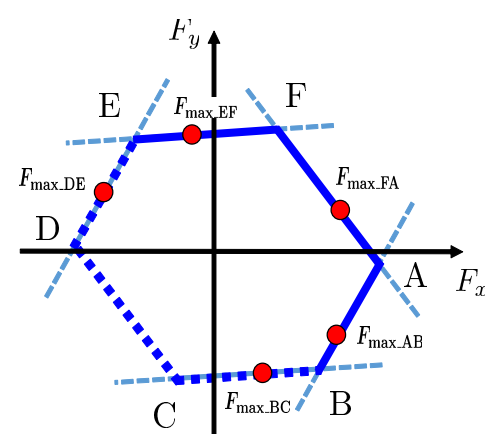

Fig. 4. Five-side measurement method

observed for a certain posture of limbs. Obtained OFD is different depending on sides without measurement. There is a large error between obtained OFD and measured actual maximum force. Even though these problems can be solved by measurement of all six sides of OFD using (3), the obtained hexagon does not satisfy the characteristics of OFD shown in (2) because length of opposite sides are not the same.

3.4 Output Force Distribution by Optimization A method to obtain an OFD similar to the hexagon by the sixside measurement under the constraint of equal length of opposite sides is proposed.

$$
\min _{d_{\mathrm{i}}} J=\sum_{\mathrm{i}}\left(D_{\text {max }_{-i}}\left(F_{x}, F_{y}\right)-d_{\mathrm{i}}\right)^{2} \ldots \ldots \ldots \ldots \ldots
$$

subject to $\overline{\mathrm{BC}}=\overline{\mathrm{EF}}$.

$\overline{\mathrm{BC}}$

$$
=\left[\begin{array}{lll}
\frac{a_{\mathrm{AB}}^{2}+1}{a_{\mathrm{BC}}-a_{\mathrm{AB}}} & \frac{-\left(a_{\mathrm{BC}}^{2}+1\right)\left(a_{\mathrm{CD}}-a_{\mathrm{AB}}\right)}{\left(a_{\mathrm{BC}}-a_{\mathrm{AB}}\right)\left(a_{\mathrm{CD}}-a_{\mathrm{BC}}\right)} & \frac{a_{\mathrm{CD}}^{2}+1}{a_{\mathrm{CD}}-a_{\mathrm{BC}}}
\end{array}\right]\left[\begin{array}{l}
d_{\mathrm{AB}} \\
d_{\mathrm{BC}} \\
d_{\mathrm{CD}}
\end{array}\right]
$$

$$
\begin{array}{ll}
\overline{\mathrm{EF}} \\
=\left[\begin{array}{lll}
\frac{-\left(a_{\mathrm{DE}}^{2}+1\right)}{a_{\mathrm{EF}}-a_{\mathrm{DE}}} & \frac{\left(a_{\mathrm{EF}}^{2}+1\right)\left(a_{\mathrm{FA}}-a_{\mathrm{DE}}\right)}{\left(a_{\mathrm{EF}}-a_{\mathrm{DE}}\right)\left(a_{\mathrm{FA}}-a_{\mathrm{EF}}\right)} & \frac{-\left(a_{\mathrm{FA}}^{2}+1\right)}{a_{\mathrm{FA}}-a_{\mathrm{EF}}}
\end{array}\right]\left[\begin{array}{c}
d_{\mathrm{DE}} \\
d_{\mathrm{EF}} \\
d_{\mathrm{FA}}
\end{array}\right]
\end{array}
$$

where $d_{\mathrm{i}}$ and $D_{\text {max } \mathrm{i}}$ are distances from origin to side i of derived OFD and side i of $\boldsymbol{F}_{\text {max_i }}$ shown in (3), respectively. Equation (5) minimizes sum of all six sides of square error of distance between OFD and the measured sides. Equation (6) shows a constraint to make length of one set of opposite sides $\mathrm{BC}$ and $\mathrm{EF}$ are the same. If the length of one set of opposite side is the same, all length of opposite sides becomes the same because all opposite sides are parallel. Equations (7) and (8) show length of sides given by a linear function of $d_{\mathrm{i}}$. Here, slopes of all sides are defined by the axis whose slope of sides BC and EF are null. The OFD derived by the optimization is called the OFD by the optimization method.

\section{Experiment}

4.1 Influence of Posture for Five-side Measurement Method Figure 5 shows definitions of joint angles and end coordinate for lower limbs. A healthy male subject exerts force by his lower limb to all directions in the plane, where gravitational force of lower limbs is subtracted from the value of a force sensor. Here, two postures are tested: posture (a)

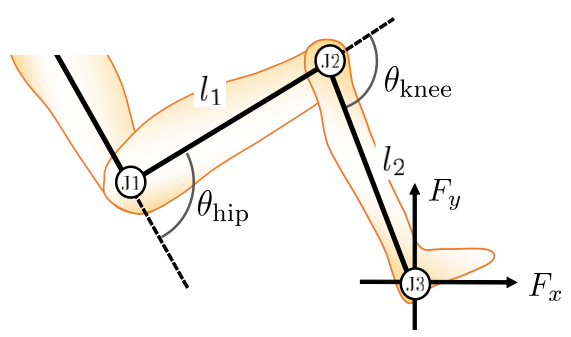

Fig. 5. Posture of lower limbs

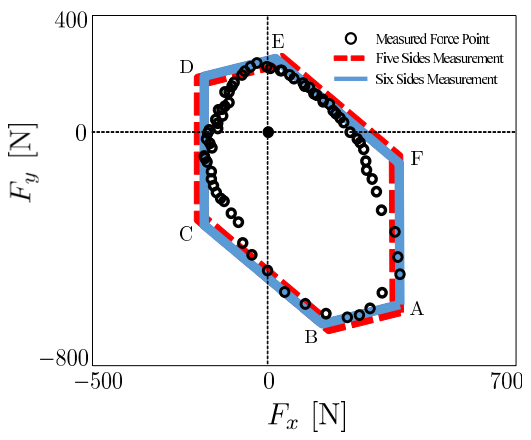

Fig. 6. OFD of posture (a)

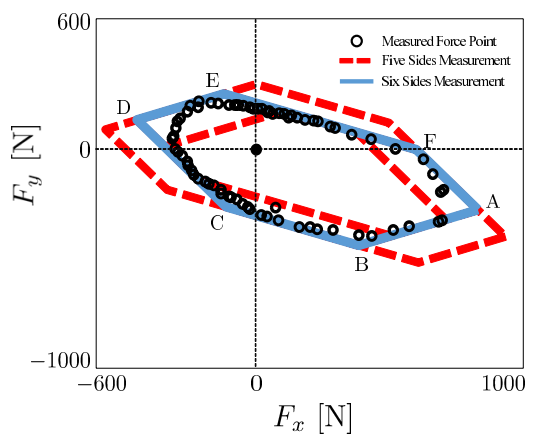

Fig. 7. OFD of posture (b)

Table 1. $R M S E_{1}$ for $O_{\mathrm{k}}$

\begin{tabular}{|c||c|c|c|c|c|c|c|}
\hline \multicolumn{1}{|c||}{} & \multicolumn{6}{c|}{ five-side measurement } & six-side measurement \\
\hline Estimated side & AB & BC & CD & DE & EF & FA & \\
\hline \hline Posture (a) & 299.5 & 271.5 & 269.8 & 337.7 & 334.3 & 277.5 & 286.0 \\
\hline Posture (b) & 368.3 & 323.3 & 455.7 & 380.1 & 476.0 & 294.4 & 256.3 \\
\hline
\end{tabular}

$\theta_{\text {hip }}=98^{\circ}, \theta_{\text {knee }}=107^{\circ}$; posture (b) $\theta_{\text {hip }}=100^{\circ}, \theta_{\text {knee }}=70^{\circ}$.

The circles $O_{\mathrm{k}}(\mathrm{k}=1,2, \cdots, 72)$ shown in Fig. 6 and Fig. 7 show maximum end force every $5^{\circ}$. The solid line hexagons are obtained from the six-side measurement. The dashed lines show estimated sides of OFD by the five-side measurement method. As shown in Fig. 7, variation of the estimated sides of OFD by the five-side measurement method increases when the knee joint stretches. Table 1 shows Root Mean Square Errors (RMSE) for two methods from $O_{\mathrm{k}}$.

$$
R M S E_{1}=\sqrt{\frac{\sum_{\mathrm{k}=1}^{72}\left(O_{\mathrm{k}}-F_{\mathrm{k}}\right)^{2}}{72}} .
$$

where $F_{\mathrm{k}}$ is the end force from OFD by the five-side measurement method or the hexagon by the six-side measurement for the same direction of $O_{\mathrm{k}}$. In posture (a), the errors of the five-side measurement method for $\mathrm{BC}, \mathrm{CD}$, and FA side estimation are small and almost the same as the error of the six-side measurement. Even though all errors of 


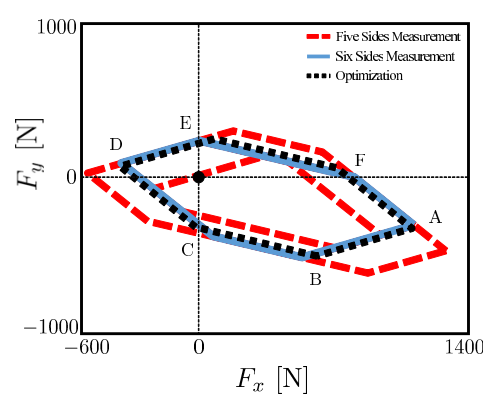

(a) Subject $\mathrm{A}$

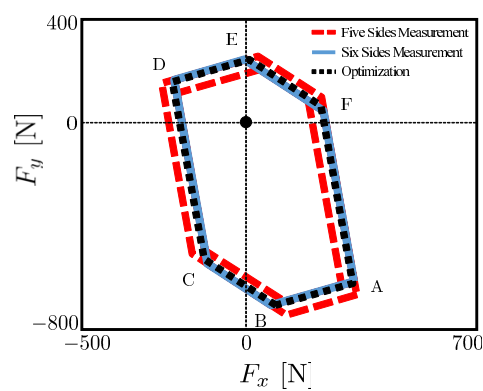

(a) Subject A

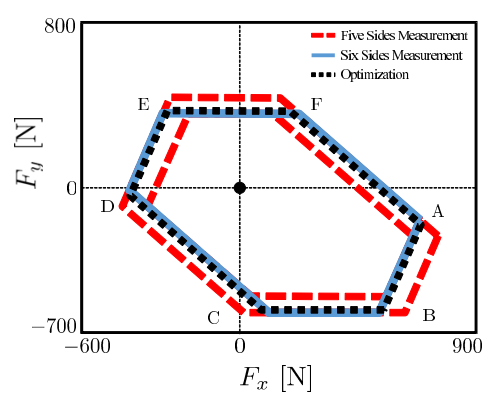

(a) Subject A

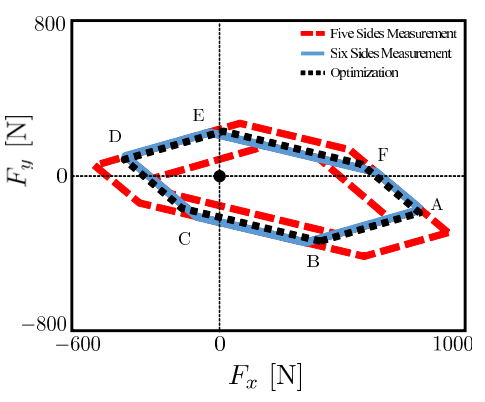

(b) Subject B

Fig. 8. Output force distribution for posture 1

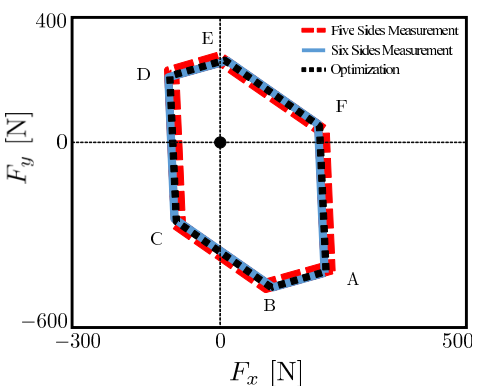

(b) Subject B

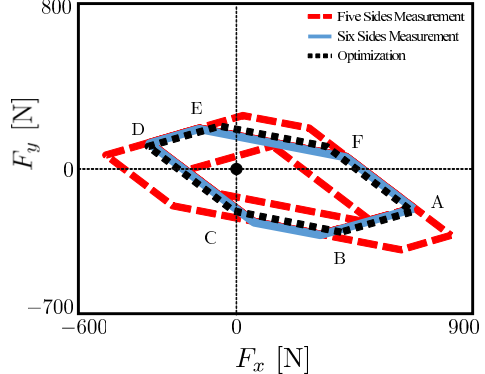

(c) Subject $\mathrm{C}$

Fig. 9. Output force distribution for posture 2

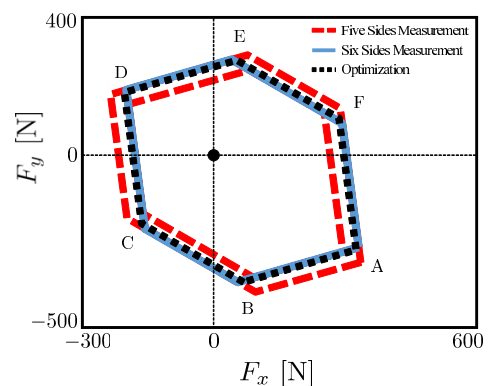

(c) Subject $\mathrm{C}$

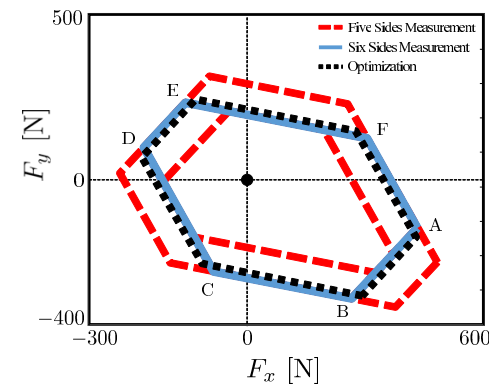

(b) Subject B

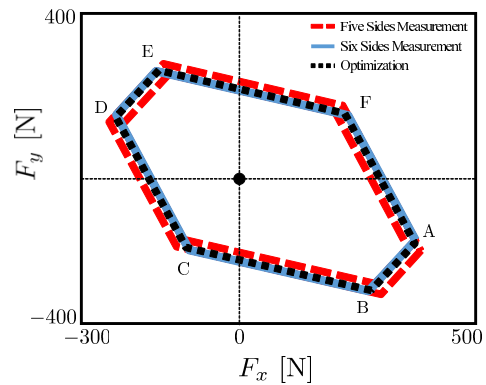

(c) Subject C

Fig. 10. Output force distribution for posture 3

Table 2. Postures of lower limbs for measurement

\begin{tabular}{|l||c|c|c|c|c|c|}
\hline \multicolumn{1}{|c||}{} & \multicolumn{2}{c|}{ Subject A } & \multicolumn{2}{c|}{ Subject B } & \multicolumn{2}{c|}{ Subject C } \\
\cline { 2 - 7 } & $\theta_{\text {hip }}$ & $\theta_{\text {knee }}$ & $\theta_{\text {hip }}$ & $\theta_{\text {knee }}$ & $\theta_{\text {hip }}$ & $\theta_{\text {knee }}$ \\
\hline \hline Posture 1 & $100^{\circ}$ & $65^{\circ}$ & $100^{\circ}$ & $67^{\circ}$ & $100^{\circ}$ & $64^{\circ}$ \\
\hline Posture 2 & $100^{\circ}$ & $102^{\circ}$ & $100^{\circ}$ & $108^{\circ}$ & $100^{\circ}$ & $104^{\circ}$ \\
\hline Posture 3 & $120^{\circ}$ & $120^{\circ}$ & $110^{\circ}$ & $120^{\circ}$ & $110^{\circ}$ & $120^{\circ}$ \\
\hline
\end{tabular}

the five-side measurement method increase when the knee joint is extended, the error of the six-side measurement is not changed so much.

4.2 Comparison of OFD of Five-side Measurement and Optimization Methods Table 2 shows three measurement postures of lower limbs for three subjects. Here, end force of lower limbs is exerted to each side of OFD.

In Fig. 8, Fig. 9, and Fig. 10, the solid lines, dashed lines, and dotted lines show hexagons by the six-side measurement, the estimated sides of OFD by the five-side measurement method, and OFD by the optimization method, respectively. Table 3 shows RMSEs for side distances between OFD and the hexagons of the six-side measurement.

$$
R M S E_{2}=\sqrt{\frac{\sum_{\mathrm{i}}\left(D_{\text {max }}\left(F_{x}, F_{y}\right)-d_{\mathrm{i}}\right)^{2}}{6}} \ldots \ldots \ldots
$$

Table 3. $R M S E_{2}$ of OFD for hexagon by six-side measurement

\begin{tabular}{|c|c||c|c|c|c|}
\hline \multicolumn{2}{|c||}{} & \multicolumn{2}{c|}{ Five-side measurement } & Optimization \\
\hline \multicolumn{2}{|c||}{ Estimated side } & AB or DE & BC or EF & CD or FA & \\
\hline \hline \multirow{3}{*}{ Posture 1 } & Subject A & 86.1 & 45.2 & 68.7 & 24.5 \\
\cline { 2 - 6 } & Subject B & 59.9 & 32.7 & 50.2 & 17.6 \\
\cline { 2 - 6 } & Subject C & 72.1 & 40.4 & 65.3 & 21.9 \\
\hline \hline \multirow{3}{*}{ Posture 2 } & Subject A & 21.2 & 14.7 & 16.7 & 6.9 \\
\cline { 2 - 6 } & Subject B & 9.3 & 7.1 & 7.7 & 3.2 \\
\cline { 2 - 6 } & Subject C & 17.8 & 13.3 & 15.5 & 6.2 \\
\hline \hline \multirow{3}{*}{ Posture 3 } & Subject A & 20.0 & 16.9 & 15.6 & 7.0 \\
\cline { 2 - 6 } & Subject B & 38.9 & 35.2 & 32.8 & 14.4 \\
\cline { 2 - 6 } & Subject C & 10.3 & 9.3 & 8.4 & 3.8 \\
\hline
\end{tabular}

Errors of OFD from the hexagons of the six-side measurement increase when knee joints are extended as shown in the posture 1 . In the posture 1 and 2 of the five-side measurement method, the error of OFD of $\mathrm{BC}$ or EF side estimation is the smallest to the other sides. In the posture 3 , the error of OFD of CD or FA side estimation is the smallest. The OFD error of the optimization method is about half of the smallest OFD error of the five-side measurement method.

4.3 Discussion In the OFD by the five-side measurement method shown in Table $3, R M S E_{2}$ difference depending 

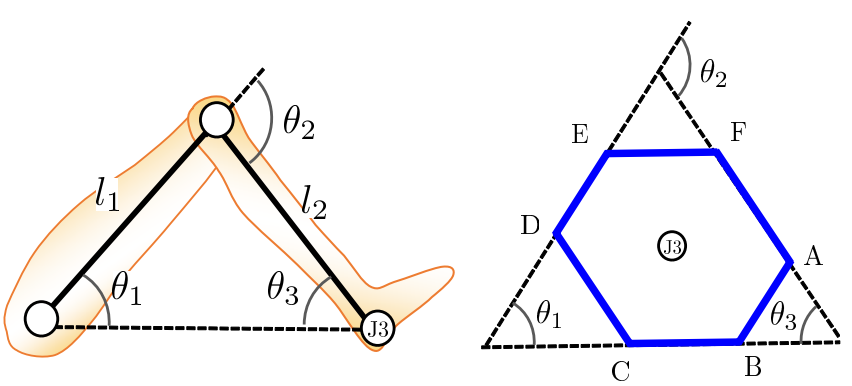

Fig. 11. Posture of lower limb and side slope of OFD

on the estimated sides is analyzed.

As shown in Fig. 11, the slope of each side of the hexagon is decided by the slope of the triangle connecting each joint of lower limbs. Table 4 shows side length deviation $\Delta L_{i}$ for the distance deviation from origin to side $i \Delta d_{i}$ derived geometrically. If the value is large, the length of the side can be changed largely by small movement of the side. Therefore, decrease of $R M S E_{2}$ is expected if the side having the largest value is estimated by the five-side measurement method.

Table 5 shows the side which has the largest value of Table 4 for similar parameters of subjects $l_{1}=0.385 \mathrm{~m}$ and $l_{2}$ $=0.475 \mathrm{~m}$. Here, the computation is possible because $\theta_{1}$ and $\theta_{3}$ are decided uniquely if $\theta_{2}, l_{1}$, and $l_{2}$ are given. Moreover, side $\mathrm{AB}$ and side $\mathrm{DE}$ are not selected because the value is smaller than others for $l_{1}<l_{2}$. This analysis coincides with the result shown in Table 3 because $\theta_{\text {knee }}=\theta_{2}$. The geometrical index is useful to decide a side without measurement for the five-side measurement method.

\section{Conclusion}

This paper deals with drawing methods of OFD to improve accuracy of maximum muscular strength derived by the theory of functionally different effective muscles.

For the measured exerted end force of a lower limb of a subject, the error of OFD of the five-side measurement method increases as the knee joint is extended. On the other hand, the error of the hexagon by the six-side measurement is not affected by knee joint variation. Therefore, this paper proposes a new OFD drawing method which solves the optimization problem to decide OFD similar to the measured hexagon by the six-side measurement while satisfying the characteristics of OFD.

In order to evaluate OFD by the five-side measurement method and the optimization method, they are applied to lower limbs of three subjects. Errors of OFD of these methods from the hexagons by the six-side measurement increase when knee joints are extended. The error of the optimization method is almost half of the smallest error of the five-side measurement method. Even though the errors of the five-side measurement method is larger than the error of the optimization method, the errors of OFD by the five-side measurement method can be made as small as possible by choosing an estimation side using the geometrically derived index.

Because the shape of measured maximum end force of lower limbs doesn't hexagonal, it influences on the result of muscular strength derived from the hexagonal OFD. It is necessary to analyze the influence or develop a new oval shape OFD drawing method and its muscular strength
Table 4. Variation of side length of OFD $\Delta L_{\mathrm{j}}$ for variation of estimated side distance $\Delta d_{\mathrm{i}}$

\begin{tabular}{|c||c|c|c|}
\hline Side & $\mathrm{i}=\mathrm{AB}$ or $\mathrm{DE}$ & $\mathrm{i}=\mathrm{BC}$ or $\mathrm{EF}$ & $\mathrm{i}=\mathrm{CD}$ or FA \\
\hline \hline$\Delta L_{\mathrm{i}}$ & $\frac{\Delta d_{\mathrm{i}}}{\tan \theta_{1}}+\frac{\Delta d_{\mathrm{i}}}{\tan \left(\pi-\theta_{2}\right)}$ & $\frac{\Delta d_{\mathrm{i}}}{\tan \theta_{1}}+\frac{\Delta d_{\mathrm{i}}}{\tan \theta_{3}}$ & $\frac{\Delta d_{\mathrm{i}}}{\tan \theta_{3}}+\frac{\Delta d_{\mathrm{i}}}{\tan \left(\pi-\theta_{2}\right)}$ \\
\hline
\end{tabular}

Table 5. Estimated side by five-side measurement method for improvement of $R M S E_{2}$ for $l_{1}=0.385 \mathrm{~m}$ and $l_{2}=0.475 \mathrm{~m}$

\begin{tabular}{|c||c|c|}
\hline & $0<\theta_{2}<113^{\circ}$ & $113^{\circ}<\theta_{2}<\pi$ \\
\hline \hline Estimated side & BC or EF & CD or FA \\
\hline
\end{tabular}

derivation method ${ }^{(9)}$. Moreover, accuracy evaluation of muscular strength for both the new method and the method in this paper are needed.

\section{Acknowledgment}

This work was supported by JSPS KAKENHI Grant Number 26350658 .

\section{References}

( 1 ) T. Oshima, T. Fujikawa, and M. Kumamoto: "Functional Evaluation of Effective Leg Muscular Strength Based on a Muscle Coordinate System Composed of Bi-articular and Mono-articular Muscles-Estimation of Functional Effective Muscular Strength from Output Force Distribution", Journal of the Japan Society for Precision Engineering, Vol.67, No.11, pp.1824-1828 (2001)

( 2 ) T. Oshima, T. Fujikawa, S. Torikai, and M. Kumamoto: "Functional Evaluation of Effective Leg Muscular Strength Based on a Muscle Coordinate System Composed of Bi-articular and Mono-articular Muscles-Estimation of Manual Wheelchair Driving Force", Journal of the Japan Society for Precision Engineering, Vol.71, No.8, pp.1041-1046 (2005)

( 3 ) T. Tajima H. Fujita, K. Sato, and Y. Nakasato: "Development of the Next Generation Steering System (Development of the Twin Lever Steering System)", 2010 SAE International, 2010-01-0993, pp.1-11 (2010)

( 4 ) T. Oshima, K. Toriumi, T. Fujikawa, and M. Kumamoto: "Functional Evaluation of Effective Leg Muscular Strength Based on a Muscle Coordinate System Composed of Bi-articular and Mono-articular Muscles-Experimental Verification of the Output Force Distribution Characteristics by Leg Muscular Training", Journal of the Japan Society for Precision Engineering, Vol.71, No.9, pp.1163-1167 (2005)

( 5 ) T. Oshima, T. Fujikawa, and M. Kumamoto: "Functional Evaluation of Effective Leg Muscular Strength Based on a Muscle Coordinate System Composed of Bi-articular and Mono-articular Muscles-Simplified Measurement Technique of Output Force Distribution", Journal of the Japan Society for Precision Engineering, Vol.67, No.6, pp.944-948 (2001)

( 6 ) S. Ishida, S. Komada, D. Yashiro, J. Hirai, and A. Nishimura: "Drawing Method of Output Force Distribution of Limbs Using Five Points Measurement", Proceedings of SICE Annual Conference, pp.2263-2265 (2013)

( 7 ) S. Ishida, S. Komada, D. Yashiro, J. Hirai, and A. Nishimura: "Drawing Method of Output Force Distribution by Using Sides Measurement", Proc. of 2014 IEE-Japan Industry Applications Society Conference, Vol.2, pp.173176 (2014)

( 8 ) S. Komada, S. Ishida, D. Yashiro, J. Hirai, and A. Nishimura: "Drawing methods of Output Force Distribution by Measurement of Sides", Proc. of IEEJ international workshop on Sensing, Actuation, and Motion CONtrol (SAMCON2015), IS5-3, pp.1-4 (2015)

( 9 ) S. Ishida, D. Yashiro, S. Komada, J. Hirai, and A. Nishimura: "Drawing Method of Output Force Distribution Using Bezier Curve and Evaluation Method of Lower Limb Muscular Strengths Based on Cosine Tuning", TokaiSection Joint Conference on Electrical, Electronics, Information, and Related Engineering, J4-2 (2014) 
Satoshi Komada (Senior Member) received the B.E., M.E., and Ph.D.

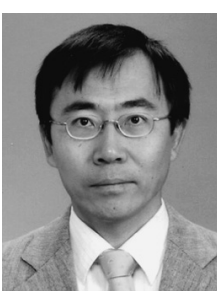
degrees from Keio University, Yokohama, Japan, in 1987, 1989, and 1994, respectively, all in electrical engineering. Since 1989, he has been with Mie University, Tsu, Japan, where he is an Associate Professor of electrical and electronic engineering. His research interests include robotics, motion control, muscular strength measurement, strengthen training, and so on.

Shohei Ishida (Non-member) received the B.E. and M.E. degrees

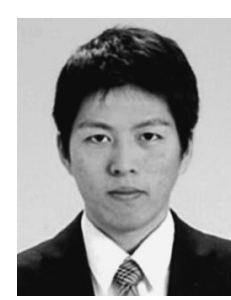
from Mie University, Mie, Japan, in 2013, and 2015, respectively, all in electrical and electronic engineering. Since 2015, he has been with Yamaha Motor Co. Ltd. His research interests include muscular strength measurement, and so on.

Daisuke Yashiro (Member) received the B.E. degree in system de-

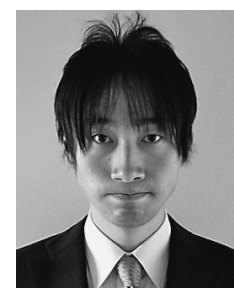
sign engineering and the M.E. and Ph.D. degrees in integrated design engineering from Keio University, Japan, in 2007, 2009, and 2010, respectively. From 2009 to 2011, he was a Research Fellow of the Japan Society for the Promotion of Science. From 2011 to 2012, he was a Research Associate at Keio University. Since 2012, he has been an Assistant Professor in the Department of Electrical and Electronic Engineering, Mie University, Japan. His research interests include motion control, haptics, real-time information processing. He is a member of the Institute of Electrical Engineers of Japan and the Institute of Electronics, Information and Communication Engineers.
Junji Hirai (Non-member) received the B.S. degree in electrical engi-

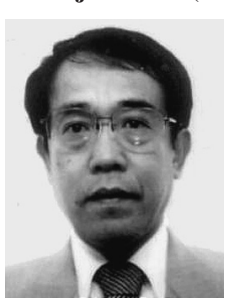
neering from the University of Tokyo, Tokyo, Japan, in 1976. He received the Ph.D. degree from Yokohama National University, Yokohama, Japan, in 1999. He joined the Yaskawa Electric Corporation, Japan, in 1982, and moved to Mie University in 2002, where he is presently a Professor in the Department of Electrical and Electronic Engineering. His fields of interest include power electronics, motion control, and robotics.

Akinobu Nishimura (Non-member) received the M.D. degree and the

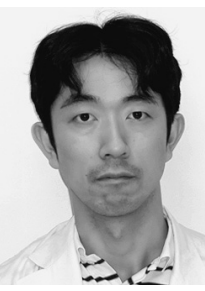
$\mathrm{Ph} . \mathrm{D}$. degree from Mie University, Tsu, Japan, in 2001 and 2010, respectively. From 2001 to 2003, he was a Resident in Orthopaedic Surgery, Department of Orthopaedic Surgery Mie University, Japan. From 2006 to 2008, he was a Graduate Research Fellow, Department of Orthopaedic Surgery, Mie University, Graduate School of Medicine, Japan. Since 2009, he has been an Assistant Professor in the Department of Orthopaedic and Sports Medicine, Mie University, Japan. His research interests orthopaedic surgery, sports medicine, preventive care. 